\title{
RhoH is a negative regulator of eosinophilopoiesis
}

\author{
Christina Stoeckle $^{1,3}$, Barbara Geering ${ }^{1,4}$, Shida Yousefi ${ }^{1}$, Saša Rožman ${ }^{1}$, Nicola Andina ${ }^{1}$, Charaf Benarafa ${ }^{2}$ and Hans-Uwe Simon ${ }^{\star, 1}$
}

Eosinophils are frequently elevated in pathological conditions and can cause tissue damage and disease exacerbation. The number of eosinophils in the blood is largely regulated by factors controlling their production in the bone marrow. While several exogenous factors, such as interleukin-5, have been described to promote eosinophil differentiation, comparatively little is known about eosinophil-intrinsic factors that control their de novo generation. Here, we report that the small atypical GTPase RhoH is induced during human eosinophil differentiation, highly expressed in mature blood eosinophils and further upregulated in patients suffering from a hypereosinophilic syndrome. Overexpression of RhoH increases, in a Rho-associated protein kinasedependent manner, the expression of GATA-2, a transcription factor involved in regulating eosinophil differentiation. In $\mathrm{RhOH}^{-/}$ mice, we observed reduced GATA-2 expression as well as accelerated eosinophil differentiation both in vitro and in vivo. Conversely, RhoH overexpression in bone marrow progenitors reduces eosinophil development in mixed bone marrow chimeras. These results highlight a novel negative regulatory role for RhoH in eosinophil differentiation, most likely in consequence of altered GATA-2 levels.

Cell Death and Differentiation (2016) 23, 1961-1972; doi:10.1038/cdd.2016.73; published online 14 October 2016

Eosinophils are short-lived effector and regulatory cells that are increasingly recognized as having key roles in the immune system, including maintenance of long-term antibody responses and defence against bacteria and viruses. ${ }^{1,2}$ On the other hand, their potentially destructive nature becomes apparent in a number of eosinophilic diseases such as hypereosinophilic syndromes (HES), diseases characterized by eosinophil-mediated tissue damage and organ dysfunction. ${ }^{3,4}$ Eosinophils contribute to disease pathology directly and indirectly by releasing cytotoxic molecules and by supporting an ongoing inflammation. ${ }^{1}$ Besides HES, eosinophilia can be observed in a wide range of infectious, allergic and autoimmune diseases, as well as in cancer. ${ }^{3,5}$ Given the increasing incidence of such diseases and the often unsatisfactory treatment options available, it is of considerable importance to better understand factors governing eosinophil biology under both normal and inflammatory conditions.

Eosinophil numbers are largely regulated by their production in the bone marrow where they differentiate from myeloid precursors. ${ }^{6}$ Lineage commitment is controlled by GATA-1, GATA-2, PU.1 and c/EBP transcription factors. For instance, mice lacking GATA-1 or c/EBP expression are devoid of eosinophils, and transduction of hematopoietic progenitor cells (HPC) with either of these genes promotes differentiation into eosinophils. ${ }^{7}$ However, the situation is not always straightforward. Lineage specification and maturation strongly depend on the relative levels of these transcription factors and their interaction partners or competitors. For example, while GATA-2 expression is required for the proper differentiation of several haematopoietic lineages, including eosinophils, ${ }^{8}$ high GATA-2 expression inhibits HPC proliferation. ${ }^{9,10}$ Similarly, overexpression of GATA-2 in progenitor cells in the presence of C/EBPa drives eosinophil differentiation, while it promotes mast cell/basophil generation in the absence of C/EBPa. ${ }^{11}$ Furthermore, while GATA-2 induces the expression of eosinophil-derived neurotoxin, it also antagonizes GATA-1-mediated expression of other eosinophil-related genes, including major basic protein. ${ }^{12-14}$ These apparently discordant findings highlight the need for a better understanding of the molecular mechanisms controlling eosinophil differentiation.

The best studied cytokines promoting eosinophil development are interleukin (IL)-3, IL-5, and granulocyte/macrophage colony-stimulating factor (GM-CSF). ${ }^{6}$ In addition to their role in eosinophilopoiesis, they also enhance eosinophil survival. ${ }^{15}$ Of these eosinophil hematopoietins, IL-5 is the most specific for the eosinophil lineage. Transgenic overexpression of $I L-5$ in mice results in eosinophilia, whereas deletion of the $I L-5$ gene strongly reduces eosinophil counts in blood and lungs following allergen challenge. ${ }^{6,16}$ Furthermore, many eosinophilic diseases are associated with increased IL-5 levels. ${ }^{3,17}$ These findings together with the knowledge of the critical role of IL-5 for eosinophil generation and survival have prompted the development of therapeutic anti-IL-5 and anti-IL-5 receptor

\footnotetext{
${ }^{1}$ Institute of Pharmacology, University of Bern, Bern, Switzerland and ${ }^{2}$ Theodor Kocher Institute, University of Bern, Bern, Switzerland

*Corresponding author: HU Simon, Institute of Pharmacology, University of Bern, Inselspital, INO-F, CH-3010 Bern, Switzerland. Tel: +41 31 632 3281 ; Fax: +41 31632 4992; E-mail: hus@pki.unibe.ch

${ }^{3}$ Current address: Novartis Institutes for Biomedical Research, Novartis Pharma AG, Basel, Switzerland

${ }^{4}$ Current address: Roche Pharma Research \& Early Development, Roche Innovation Center Basel, Basel, Switzerland

Abbreviations: ECP, eosinophil cationic protein; EDN, eosinophil-derived neurotoxin; FACS, fluorescence-activated cell sorting; Flt3L, Fms-related tyrosine kinase 3 ligand; G-CSF, granulocyte colony-stimulating factor; GM-CSF, granulocyte/macrophage colony-stimulating factor; HES, hypereosinophilic syndrome; HPC, hematopoietic progenitor cells; 4-OHT, 4-hydroxytamoxifen; IL, interleukin; MEK, mitogen/extracellular signal-regulated kinase; PBMC, peripheral blood mononuclear cells; PCR, polymerase chain reaction; PI3K, phosphoinositide 3-kinase; ROCK, Rho-associated protein kinase; SCF, stem cell factor; STAT, signal transducer and activator of transcription; WT, wild-type

Received 11.1.16; revised 16.6.16; accepted 27.6.16; Edited by P Salomoni; published online 14 October 2016
} 
a

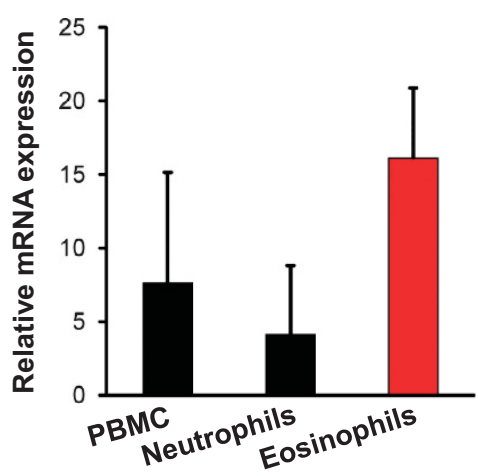

C
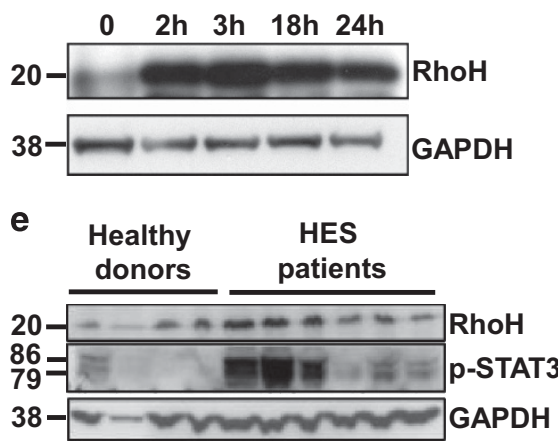

b

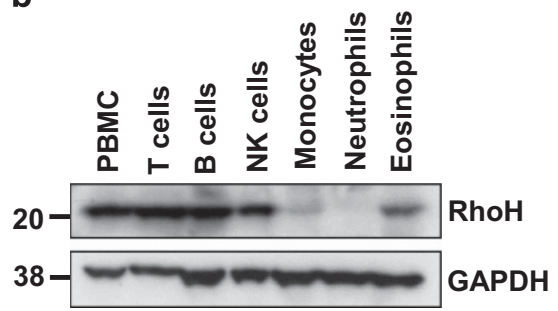

d

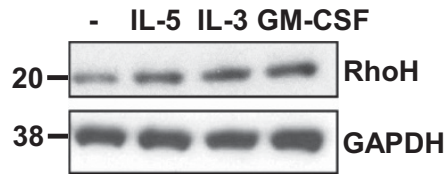

f

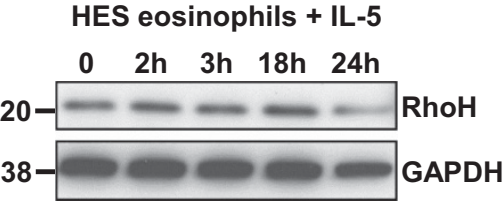

Figure $1 \mathrm{RhoH}$ expression in peripheral blood eosinophils. Leukocyte subsets were isolated from peripheral blood and RhoH expression was measured by qPCR (a) or immunoblotting (b). Representative immunoblots of eosinophils from healthy donors stimulated with $10 \mathrm{ng} / \mathrm{ml}$ IL-5 (c) or $10 \mathrm{ng} / \mathrm{ml}$ of IL-5, IL-3 or GM-CSF for $3 \mathrm{~h}$ (d), freshly isolated eosinophils from healthy donors or HES patients (e), or IL-5 stimulated eosinophils from HES patients (f), are presented. Values in panel (a) represent means $+/-$ S.D. Data in panels $(\mathbf{b}-\mathbf{f})$ are representative for at least three independent experiments

antibodies, which are currently being investigated in eosinophilic disorders. ${ }^{1,18}$

$\mathrm{RhoH}$ belongs to the family of small GTPases that are involved in diverse cellular processes, including signalling, vesicle transport, migration, proliferation and differentiation. In contrast to most family members, RhoH is GTPase deficient and, therefore, its activity is assumed to be regulated at the level of protein expression rather than by switching between active (GTP-bound) and inactive (GDP-bound) forms. ${ }^{19} \mathrm{RhoH}$ is exclusively expressed in the hematopoietic system and is mutated in a number of lymphomas and leukemias, but the mechanisms by which dysregulated $\mathrm{RhoH}$ contributes to malignancy remain unclear.

With respect to its physiological functions, $\mathrm{RhoH}$ is involved in T-cell receptor (TCR) signalling, where it regulates the subcellular localization of ZAP70 and LCK. ${ }^{20-22}$ Following TCR stimulation, RhoH is degraded. ${ }^{23} \mathrm{RhoH}^{-1}$ mice have reduced $\mathrm{T}$-cell numbers due to a failure of positive selection, and impaired peripheral T-cell responses. ${ }^{21,24}$ This phenotype is similar to that of two recently described natural $\mathrm{RhoH}$ deficient siblings, in which impaired T-cell function resulted in susceptibility to certain viral infections. ${ }^{25}$ In mast cells, RhoH is critical for FceRl-dependent signal transduction. ${ }^{26} \mathrm{RhoH}$ has also been found to negatively regulate several processes, including T-cell adhesion and migration, stem cell proliferation and homing, ${ }^{27} \mathrm{IL}-3$ signalling in a $\mathrm{B}$ cell line ${ }^{28}$ and leukotriene production in neutrophils, ${ }^{29}$ but its function in eosinophils is unknown.

In this study, we report that $\mathrm{RhoH}$ deficiency promotes, and $\mathrm{RhoH}$ overexpression reduces, eosinophil differentiation in vitro and in vivo. Moreover, $\mathrm{RhoH}$ induces the developmentally important transcription factor GATA-2 through a Rho-associated protein kinase (ROCK)-dependent mechanism. In addition, $\mathrm{RhoH}$ appears to promote cellular quiescence in a manner similar to what has been reported for GATA-2. ${ }^{10}$ Furthermore, our data suggest that IL-5 induces $\mathrm{RhoH}$ in immature eosinophils, a pathway that also remains functional following maturation. Taken together, our data suggest that IL-5 not only drives eosinophil differentiation, but also induces an inhibitory pathway limiting this process in which $\mathrm{RhoH}$ has a major role.

\section{Results}

RhoH is expressed in human eosinophils and upregulated upon IL-5 stimulation. To investigate whether RhoH is expressed in eosinophils under physiological conditions, we isolated eosinophils from peripheral blood of healthy donors and found that eosinophils expressed higher levels of $\mathrm{RhoH}$ mRNA than peripheral blood mononuclear cells (PBMC) or neutrophils from the same individuals (Figure 1a). To examine $\mathrm{RhoH}$ protein expression in more detail, we isolated different leukocyte subsets for immunoblot analysis. We 

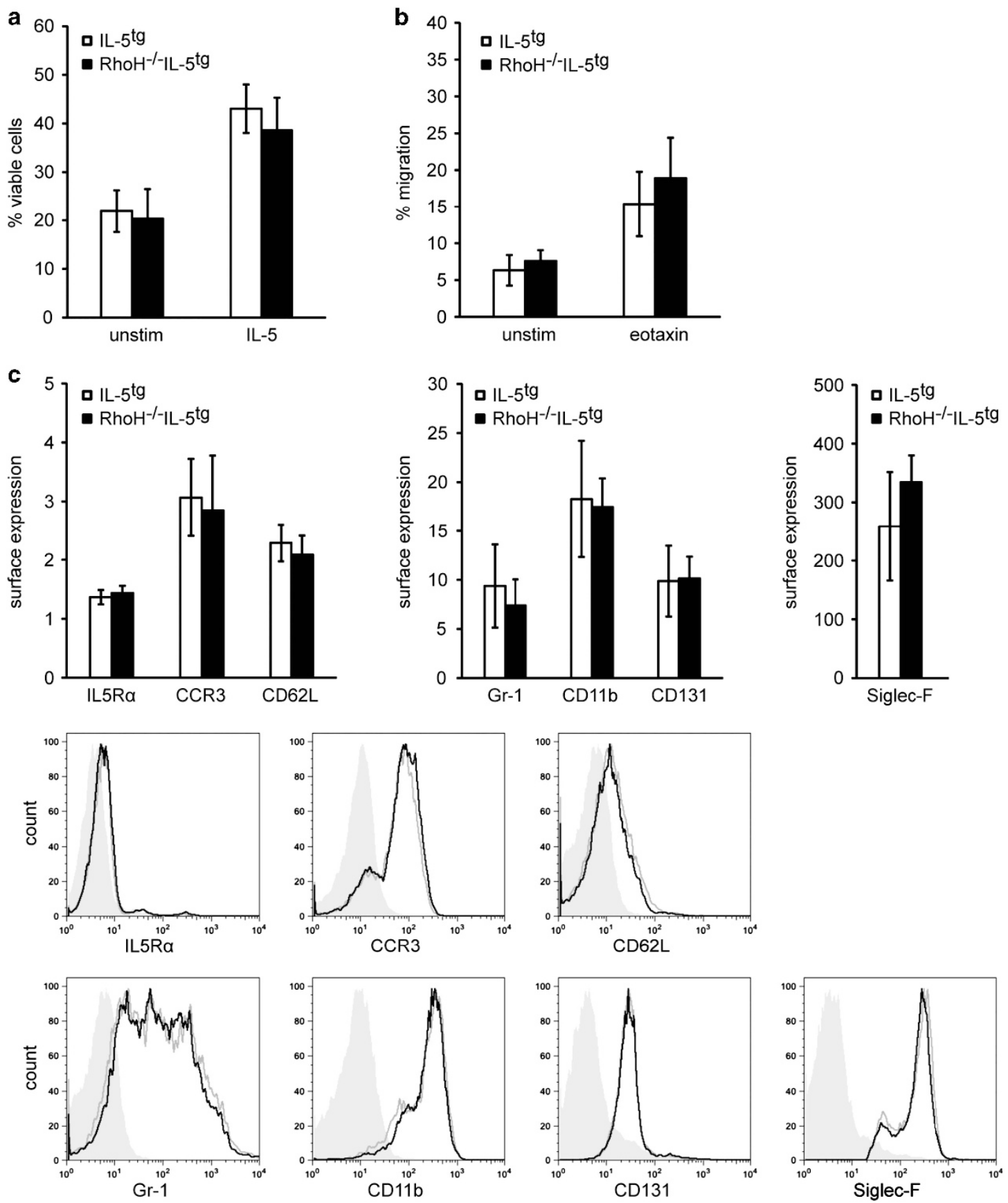

Figure 2 Absence of $\mathrm{RhoH}$ had no effect on viability, migration and immunophenotype of eosinophils obtained from IL-5 overexpressing mice. Eosinophils were isolated from the spleens of $I L 5^{t g}$ mice or $R h \mathrm{H}^{-1} / L 5^{t g}$ mice and analyzed in vitro. (a) Viability after $48 \mathrm{~h}$ in culture. (b) Migration in the absence of a stimulus or after $100 \mathrm{ng} / \mathrm{ml}$ eotaxin treatment. (c) Expression of the indicated surface molecules of freshly isolated eosinophils. Values represent means $+/-$ S.D. ( $n \geq 4)$. Representative FACS plots are shown. Filled gray: isotype control, black line: $R h O H^{-1} I L 5^{t g}$, gray line: $I L 5^{t g}$ eosinophils

observed that eosinophils and NK cells express RhoH. In addition, in agreement with the previous observations, ${ }^{23}$ $\mathrm{RhoH}$ protein was present in T and B cells, but was hardly detectable in monocytes and neutrophils (Figure 1b).

We next investigated the regulation of $\mathrm{RhoH}$ by eosinophil hematopoietins. $\mathrm{RhoH}$ was rapidly upregulated in eosinophils in response to IL-5 (Figure 1c), IL-3 and GM-CSF (Figure 1d). Of note, the magnitude of the response differed considerably between donors $3 \mathrm{~h}$ after stimulation (Figures 1c and d), ranging from 1.5 -fold to more than 10 -fold induction, probably reflecting differences in intrinsic responsiveness and/or degrees of pre-activation. The reduction of $\mathrm{RhoH}$ signal at later time points is mirrored by a reduction in GAPDH signal and is likely the result of a loss of cell viability.

To address the in vivo relevance of these findings, we assessed $\mathrm{RhoH}$ expression in eosinophils from healthy 
a

HL-60-RhoH

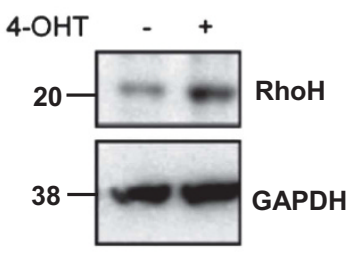

HL-60-GFP

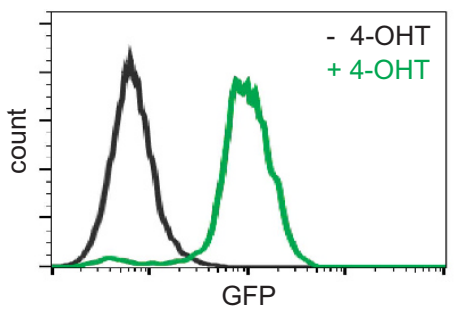

b

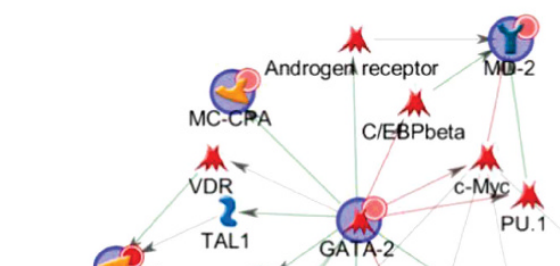

Carbonic anthydrase II 2

$\underbrace{\text { ZTAT3 GATA-3 }}_{\text {Neuropilin-2 }}$

NRCAM

GATA-1

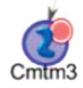

transcription factor

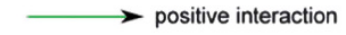

2 binding protein

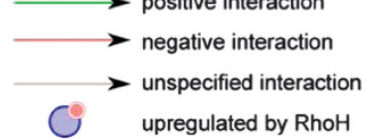

$Y$ receptor upregulated by $\mathrm{RhoH}$

C
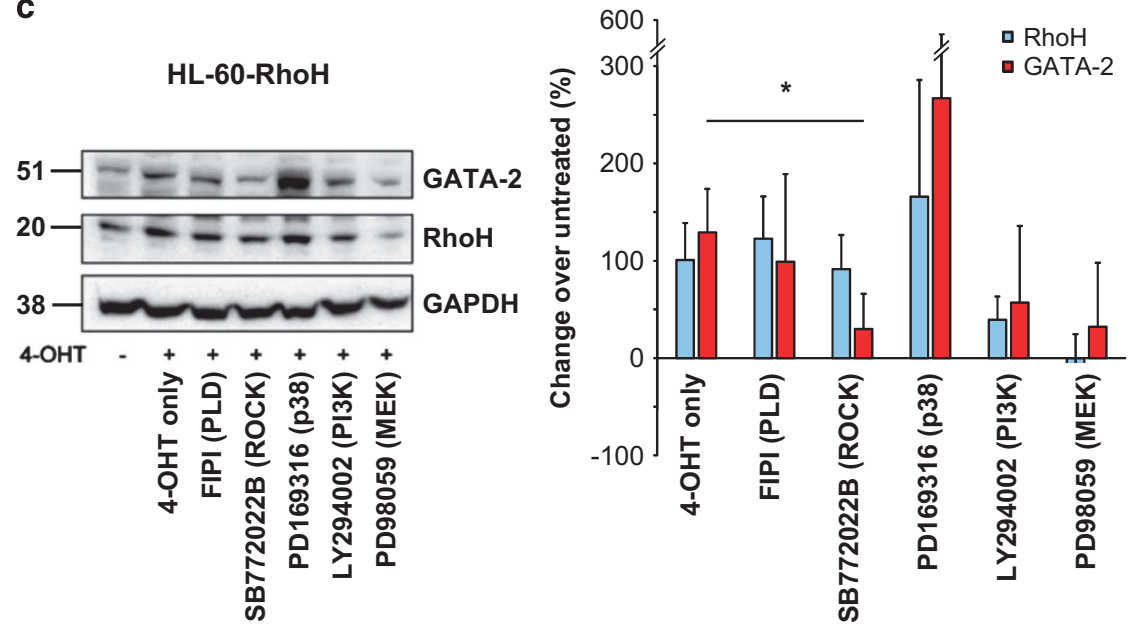
donors and patients with HES, a condition frequently characterized by overproduction of IL-5 or IL-3 by T cells or other cells. $^{3,17}$ We found that in eosinophils from some HES patients, $\mathrm{RhoH}$ was upregulated (Figure 1e). Notably, increased $\mathrm{RhoH}$ expression was accompanied by phosphorylation of signal transducer and activator of transcription 3 (STAT3). Why STAT3 phosphorylation varied considerably among HES patients is unclear, as the eosinophilia in all cases was likely driven by at least one of the eosinophil hematopoietins IL-3, IL-5 or GM-CSF and none of these patients had evidence for the presence of an eosinophilic leukemia. Therefore, we speculate that STAT3 phosphorylation is a dynamic process and depends not only on extracellular cytokine concentrations at a given time point. Nevertheless, the increased STAT3 phosphorylation levels point to the possibility that increased RhoH levels in HES patients might have been caused by in vivo exposure to at least one eosinophil hematopoietin. This notion was further substantiated by the finding that eosinophils derived from HES patients failed to further upregulate $\mathrm{RhoH}$ in response to in vitro stimulation with IL-5 (Figure 1f). Mature eosinophils from RhoH knockout mice exhibit no
abnormalities regarding life span, surface phenotype or migration. To determine the functional role of $\mathrm{RhoH}$ in resting and activated eosinophils, we attempted to overexpress $\mathrm{RhoH}$ in primary human eosinophils. However, primary granulocytes are notoriously difficult to transfect. As we have previously observed that the use of lentiviruses is not suitable for overexpressing proteins in granulocytes, ${ }^{30}$ we tested the nucleofection approach reported by Xu et al. ${ }^{31}$ for mouse neutrophils. Using a GFP control construct, we observed GFP expression in up to $40 \%$ of eosinophils (Supplementary Figure S1a). However, we found that the vast majority of eosinophils died during the procedure and surviving eosinophils showed strong signs of nonspecific activation, such as downregulation of the IL-5/IL-3/GM-CSF common $\beta$-chain ((CD131); Supplementary Figure $S 1 b)$. This is in agreement with a recent publication using human neutrophils and an identical protocol. ${ }^{32}$

To circumvent the difficulties experienced with manipulating RhoH levels in human eosinophils, we crossed $\mathrm{RhoH}^{-1}$ mice to mice carrying the $I L-5$ transgene. $I L-5^{t g}$ mice have increased levels of eosinophils, allowing their isolation in larger numbers. ${ }^{16}$ Eosinophils from $\mathrm{RhoH}^{-1} / \mathrm{L}-5^{\text {tg }}$ mice did not show any obvious alterations in cellular viability, migratory behaviour (spontaneous or eotaxin-mediated), surface immunophenotype (IL-5Ra, CCR3, CD62L, Gr-1, CD11b, CD131) or spontaneous and induced cytokine (IL-4, IL-6, IL-13) production compared with eosinophils from $I L-5^{t g}$ mice (Figure 2 and data not shown).
RhoH induces GATA-2 expression. As genetic modification of human eosinophils was problematic and functional screening with mouse eosinophils did not uncover a role for $\mathrm{RhoH}$ in mature eosinophils, we lentivirally transduced the eosinophilic HL-60c15 line with 4-hydroxytamoxifen (4-OHT)inducible constructs coding for $\mathrm{RhoH}(\mathrm{HL}-60-\mathrm{RhoH})$ or GFP $(\mathrm{HL}-60-\mathrm{GFP})^{33}$ as a control. Induction of $\mathrm{RhoH}$ or GFP by 4-OHT was confirmed by western blot or fluorescenceactivated cell sorting (FACS) analysis (Figure 3a). Using microarrays, gene expression of untreated HL-60-RhoH cells was compared with that of $\mathrm{HL}-60-\mathrm{RhoH}$ cells in which $\mathrm{RhoH}$ had been induced. To exclude genes that were induced by 4-OHT alone, 4-OHT-treated HL-60-GFP cells were also included in the analysis. Among the genes found to be specifically upregulated by $\mathrm{RhoH}$, most had no known functions in eosinophil physiology (Supplementary Table S1). To further explore the patterns underlying these data, we performed network and Gene Ontology (GO) analysis using Metacore (Figure $3 \mathrm{~b}$ and Supplementary Figure S2). The resulting interaction network centred on GATA-2, a transcription factor involved in eosinophil differentiation. Similarly, the GO processes associated with the data included 'eosinophil differentiation' and 'eosinophil fate commitment', and a prominent component of these GO processes was again GATA-2, suggesting that $\mathrm{RhoH}$ might influence eosinophil differentiation by altering GATA-2 expression.

A link between $\mathrm{RhoH}$ and GATA-2 has not been described previously. To investigate which molecular pathways might be involved in RhoH-induced GATA-2 upregulation, we treated $\mathrm{HL}-60-\mathrm{RhoH}$ cells with inhibitors for different signalling pathways before induction (Figure $3 \mathrm{c}$ ). $\mathrm{RhoH}$ induction led to upregulation of GATA-2 at the protein level, confirming the microarray data. P38 inhibition resulted in GATA-2 upregulation, but this was not statistically significant. MEK and PI3K inhibition lead to reduced GATA-2 induction, but RhoH also failed to be upregulated under those conditions, suggesting that the inhibitors act upstream of $\mathrm{RhoH}$ in this system and that lack of GATA-2 upregulation is likely a consequence of lack of $\mathrm{RhoH}$ induction. Interestingly, of the pharmacological inhibitors tested, only the ROCK inhibitor SB772022B prevented GATA-2 upregulation without affecting $\mathrm{RhoH}$ induction (Figure $3 \mathrm{c}$ ), indicating that ROCK is a downstream effector of $\mathrm{RhoH}$ and mediates $\mathrm{RhoH}$-induced GATA-2 upregulation.

RhoH is upregulated during differentiation of human eosinophils. As the microarray data suggested a role of $\mathrm{RhoH}$ in eosinophilopoiesis, we investigated whether $\mathrm{RhoH}$ would regulate this process. We found that $\mathrm{RhoH}$ expression was low in undifferentiated $\mathrm{HL}-60 \mathrm{c} 15$ cells, but was upregulated during differentiation into eosinophil-like cells

Figure 3 GATA-2 induction in RhoH overexpressing cells. (a) Induction of RhoH (immunoblot) or GFP expression (FACS) by 4-OHT in transduced cells. (b) Network analysis of genes selectively regulated by $\mathrm{RhoH}$ as determined by microarray analysis. Input genes (i.e., those regulated by $\mathrm{RhoH}$ ) are marked with blue circles, interactions with arrows (green: positive regulatory interactions, red: negative regulatory interactions, gray: unspecified interactions). Small red circles indicate upregulation. Microarray analysis was performed with three biological replicates per condition. (c) Regulation of GATA-2 protein by RhoH and ROCK. HL-60-RhoH cells were induced to overexpress RhoH with 4-OHT in the presence or absence of different signalling inhibitors. The molecular target of each inhibitor is given in brackets. A representative immunoblot and quantification of the results are shown $(n=3)$. Expression of RhoH and GATA-2 were normalized to that of GAPDH, and then to that of the untreated sample. The quantification graph shows the change in expression relative to the untreated control. Values represent means $+/-$ S.D. ${ }^{*} P<0.05$ for GATA-2 expression compared with the 4-OHT-treated sample 
a

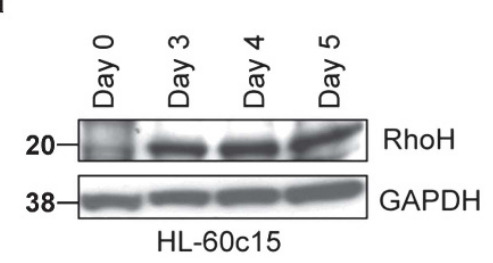

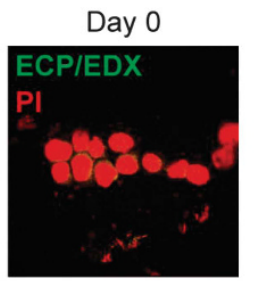

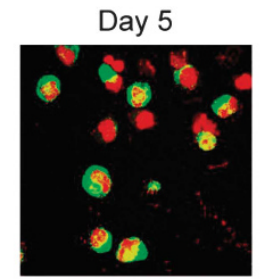

b

Immature human bone marrow granulocytes

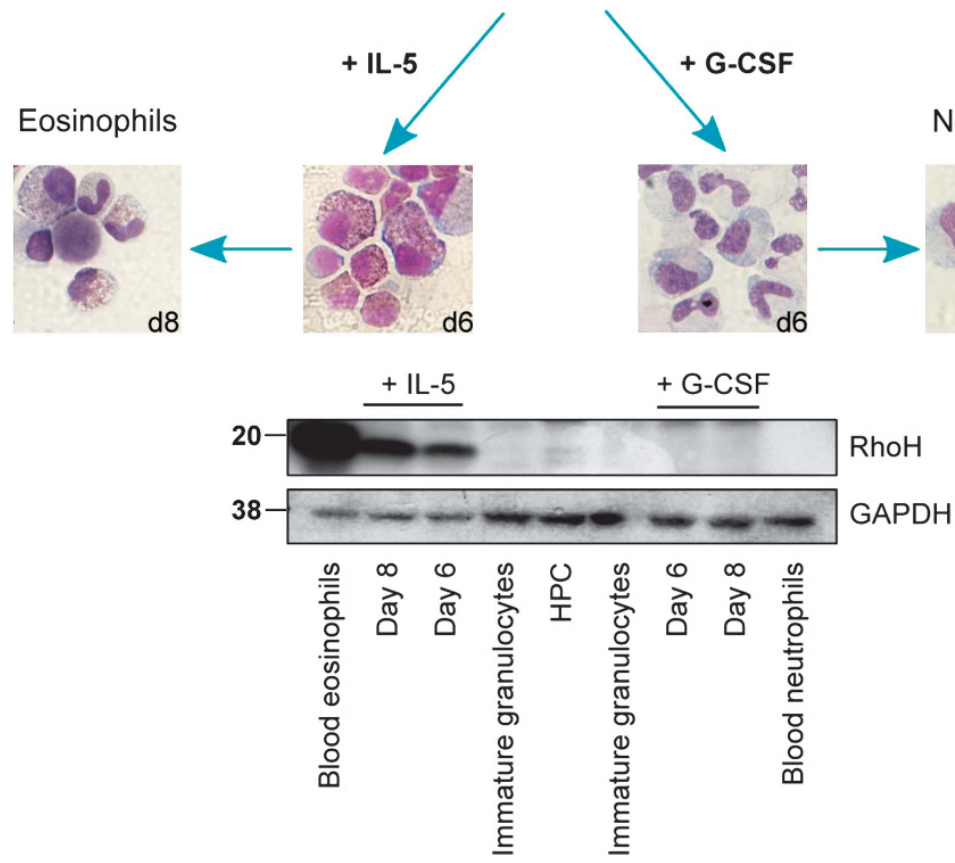

Figure 4 RhoH expression in differentiating eosinophils. (a) HL-60c15 cells were differentiated into eosinophil-like cells for the indicated number of days. Left: Immunoblot. $\mathrm{RhoH}$ is induced during differentiation. Right: Immunofluorescence. The expression of eosinophil cationic protein/eosinophil-derived neurotoxin ECP/EDN is induced (original magnification $\times 630$ ), confirming eosinophil differentiation. (b) Immature granulocytes isolated from human bone marrow were differentiated into mature neutrophils by addition of G-CSF, or eosinophils by addition of IL-5 for 8 days. Differentiation and maturation were confirmed by morphological assessment of Diff-Quik-stained cytospins (original magnification $\times 630$ for all panels). RhoH expression was determined in mature blood eosinophils and neutrophils, hematopoietic progenitor cells (HPC) and immature granulocytes isolated from human bone marrow, as well as for in vitro differentiated eosinophils or neutrophils

(Figure 4a). To further substantiate these data in a more physiological system, we isolated HPC and immature myeloid cells from human bone marrow. Immature myeloid cells were differentiated into mature neutrophils by addition of G-CSF, or into eosinophils by addition of IL-5, and analyzed by immunoblot (Figure 4b). RhoH was barely detectable in HPC and was undetectable in immature myeloid cells, differentiating and mature neutrophils. Differentiation into eosinophils, on the other hand, resulted in strong upregulation of $\mathrm{RhoH}$, explaining the relatively high $\mathrm{RhoH}$ levels in mature peripheral blood eosinophils.

$\mathrm{RhoH}^{-}$mice have increased eosinophil numbers in peripheral blood and bone marrow. To investigate a potential role of $\mathrm{RhoH}$ in eosinophil differentiation in vivo, we analyzed the peripheral blood and bone marrow of $\mathrm{RhoH}^{-1-}$ and wild-type (WT) mice, and found that absolute eosinophil counts were clearly increased in the peripheral blood of knockout mice (Figure 5a). Similarly, eosinophils were more abundant in the bone marrow of $\mathrm{RhoH}^{--}$mice
(Figure 5b), suggesting that the elevated number of eosinophils in peripheral blood was due to increased de novo production. Unexpectedly, we also found that eosinophils in the bone marrow of $\mathrm{RhoH}^{-1}$ mice were more mature than the WT as assessed by morphological criteria (Figure 5c). Mature eosinophils differ in their surface phenotype from their immature counterparts, having upregulated the eotaxin receptor CCR3 and downregulated CD62L. ${ }^{34}$ In agreement with the morphological data, we observed increased CCR3 and decreased CD62L expression in the absence of $\mathrm{RhoH}$, confirming a more mature phenotype (Figure $5 \mathrm{~d}$ ).

RhoH overexpression leads to reduced eosinophil differentiation in mixed bone marrow chimera. To further substantiate the effects of $\mathrm{RhoH}$ on eosinophil differentiation in vivo, we reconstituted the hematopoietic compartment of lethally irradiated $I L-5^{t g}$ mice with a $1: 1$ mixture of hematopoietic stem and progenitor cells (HSPCs) transduced with either a $\mathrm{RhoH}$ overexpressing or control lentivirus vector (Supplementary Figure S3a, b). Four weeks post transfer, 
a

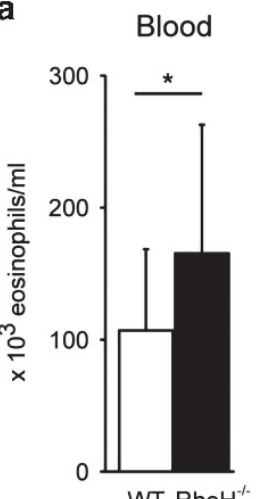

c

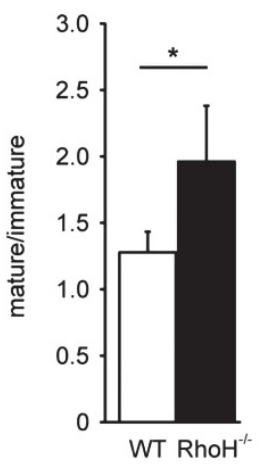

d

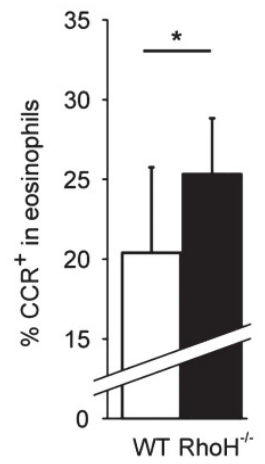

b
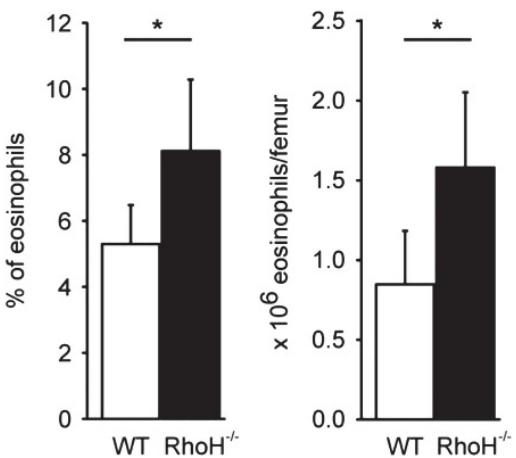

Bone marrow

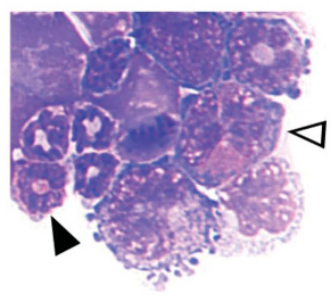

$\triangle$ immature eosinophil

mature eosinophil

Bone marrow

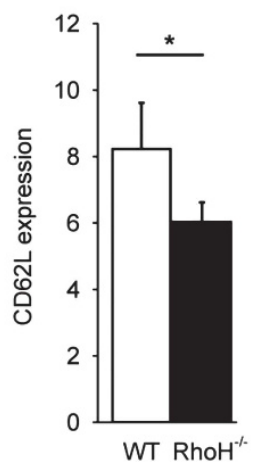

Figure 5 Increased eosinophils and eosinophil maturity in the absence of RhoH in vivo. Eosinophil numbers in blood (a) and bone marrow (b) of WT and $\mathrm{RhoH}^{-1}$ mice. Cell differentials were determined by Diff-Quik staining and microscopy. (c) Ratio mature/immature eosinophils in freshly isolated bone marrow of WT and knockout mice. Filled triangle: mature eosinophil, open triangle: immature eosinophil (original magnification $\times 630$ ). (d) Flow cytometry. Percentage of $\mathrm{CCR} 3^{+}$and level of CD62L expression on eosinophils in freshly isolated bone marrow. $(n \geq 4)$. ${ }^{*} P<0.05$

blood leukocyte were typically $95 \%$ of donor origin (Figure 6a; Supplementary Figure S3c and d). The remaining 5\% cells from the recipient mice were principally $T$ cells, which sustained high levels of IL-5 as indicated by a substantial blood eosinophilia in most mice (Figure $6 b$ ). The relative chimerism of donor-derived leukocytes revealed a highly significant skewing in favor of eosinophils originating from CD45.2 progenitors transduced with control lentivirus at the expense of eosinophils from $\mathrm{RhoH}$ overexpressing
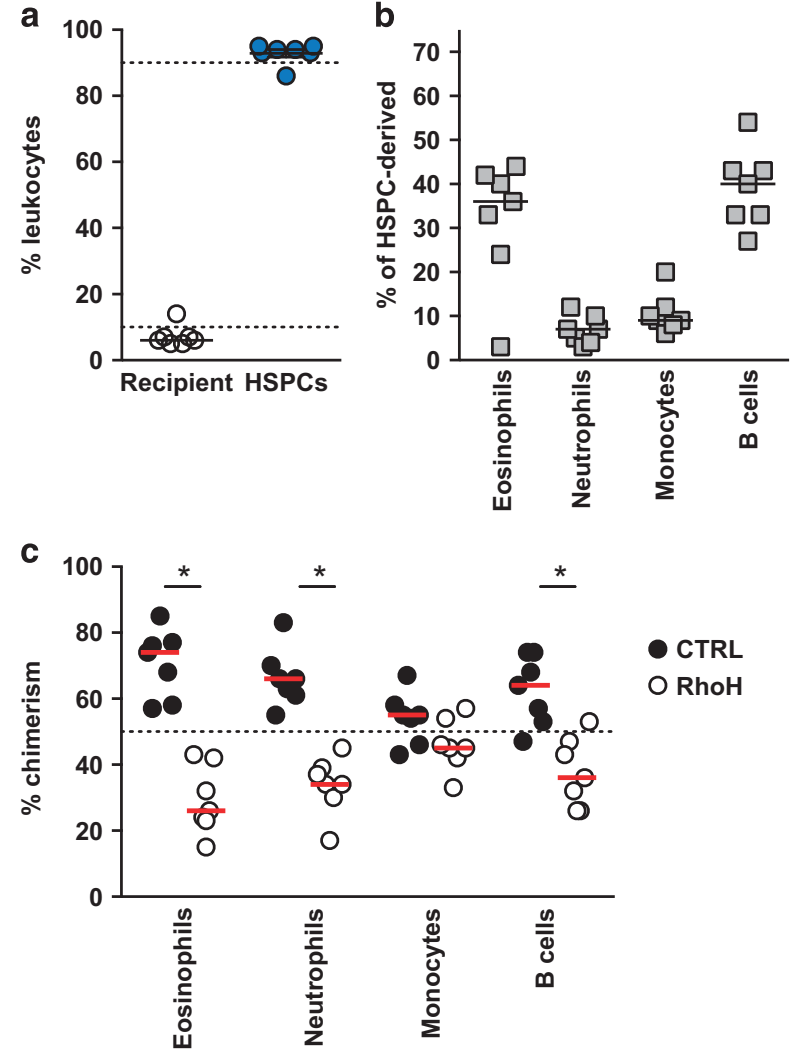

Figure $6 \mathrm{RhoH}$ overexpression in HSPCs of mixed bone marrow chimeras. (a) Percentage of donor-derived and HSPC-derived blood leukocytes 4 weeks post irradiation and HSPC transfer. (b) Percentage of blood leukocyte subsets from HSPC-derived cells. (c) Relative percentage of $\mathrm{RhoH}$ overexpressing and control HSPC-derived cells for each leukocyte subset. ${ }^{*} P<0.01$

progenitors (CD45.1). Of note, a similar, although less intense skewing, was observed in neutrophils and B cells, but not monocytes (Figure 6c; Supplementary Figure S4). At 4 weeks post transfer, most circulating cells are derived from progenitors rather than stem cells. A similar analysis performed at 8 weeks post transfer showed that $\mathrm{RhoH}$ overexpression affected all leukocyte subsets in blood and bone marrow (Supplementary Figure S3e and S5). In our competitive system, the early (4 weeks) skewing of eosinophils and neutrophils, but not monocytes, suggests that $\mathrm{RhoH}$ has a strong negative effect on some, but not all lineage-committed progenitors, including eosinophils. The results at 8 weeks are consistent with previous reports showing that $\mathrm{RhoH}$ is a broad negative regulator of hematopoietic stem and/or uncommitted progenitor cell engraftment. ${ }^{27}$ As a cautionary note, the latter results may be due in part to allelic differences in the CD45 locus, which was reported to give a competitive advantage to CD45.2 HSPCs in long-term engraftment studies (>12 weeks). ${ }^{35-37}$ However, the extent of skewing observed in eosinophils compared with B cells and neutrophils 4 weeks after transfer more strongly supports an effect of $\mathrm{RhoH}$ overexpression in eosinophil progenitors than a general effect of the CD45 allele on HSPCs. 
a

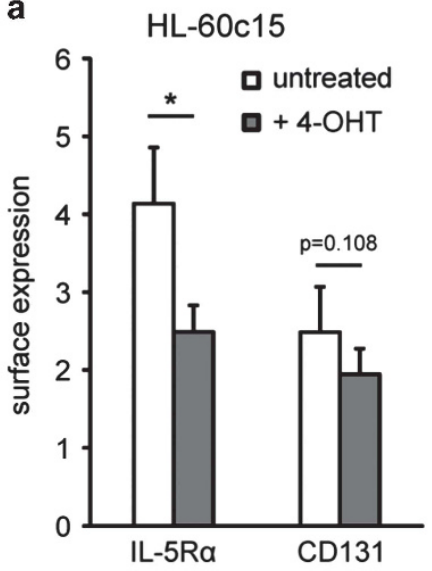

b

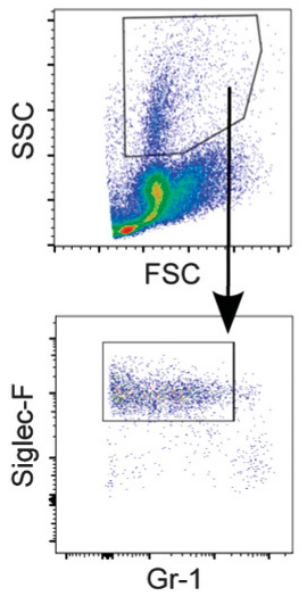

Bone marrow eosinophils

C
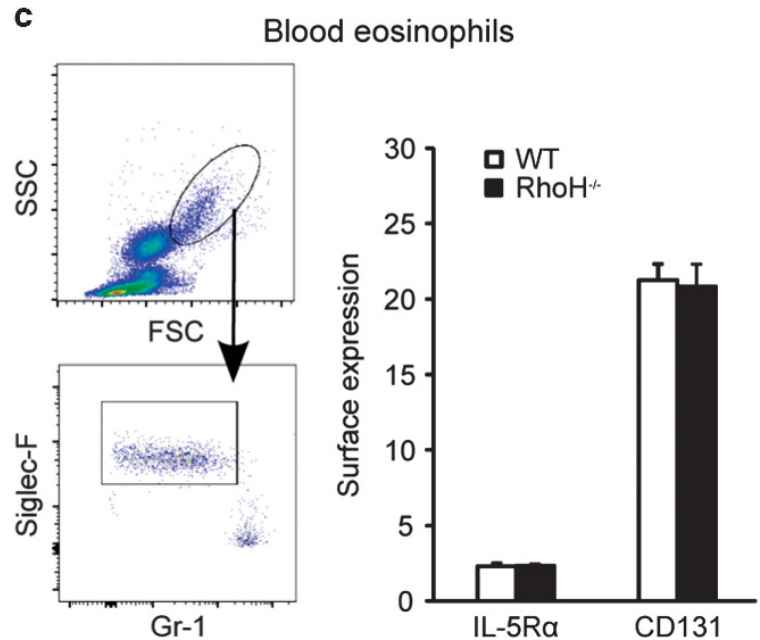

Figure 7 RhoH does not affect IL-5 receptor expression on primary mouse eosinophils. (a) Expression of IL-5R $\alpha$ and IL-5R $\beta$ (CD131) in HL-60-RhoH cells induced to overexpress RhoH with 4-OHT. (b) Gating strategy and expression of IL-5 receptor chains on freshly isolated mouse bone marrow eosinophils of WT and $\mathrm{RhoH}^{-/-}$mice. (c) Gating strategy and expression of IL-5 receptor chains on freshly isolated mouse blood eosinophils of WT and $\mathrm{RhOH}^{-1}$ mice. Values represent means $+/-$ S.D. $(n=4) .{ }^{*} P<0.05$
RhoH does not affect IL-5R expression in primary eosinophils. $\mathrm{RhoH}$ has been reported to downregulate the alpha chain of the IL-3 receptor (CD123) in a B cell line, ${ }^{28}$ diminishing IL-3 signalling. As eosinophil levels are mainly regulated through the closely related IL-5, and we had observed upregulation of $\mathrm{RhoH}$ in response to $\mathrm{IL}-5$ in eosinophils (Figure 1), we determined if $\mathrm{RhoH}$ can downregulate the IL-5 receptor in eosinophils. Although surface expression of the IL-5Ra (CD125) and $\beta$-chain (CD131) were reduced following $\mathrm{RhoH}$ induction in $\mathrm{HL}-60 \mathrm{c} 15$ cells (Figure 7a), we observed no difference in IL-5R expression on bone marrow or peripheral blood eosinophils between WT and $\mathrm{RhoH}^{-1}$ mice (Figures $\mathrm{7b}$ and c), similar to the findings with transgenic animals (Figure 2). This suggests that elevated eosinophil numbers in the absence of $\mathrm{RhoH}$ are not due to an altered IL-5 receptor expression in vivo.

Lack of RhoH results in increased eosinophil differentiation in vitro and reduced GATA-2 expression in bone marrow. To corroborate our findings that $\mathrm{RhoH}$ deficiency promotes de novo eosinophil differentiation, we employed a cell culture model to differentiate eosinophils from mouse bone marrow. ${ }^{38}$ Using this model, we observed that eosinophil differentiation is indeed enhanced in the absence of $\mathrm{RhoH}$ (Figure 8). Importantly, although the percentage of eosinophils was higher in the bone marrow of $\mathrm{RhOH}^{-}$ mice at the beginning of the culture, at day 6 virtually no eosinophils could be detected in either knockout or WT cultures, excluding the possibility that the differences observed were due to eosinophils surviving through the culture period. Newly differentiating eosinophils were discernible from day 8 onwards and subsequently developed into mature cells. This process was accelerated in the absence of RhoH as shown by increased eosinophil numbers (Figures $8 \mathrm{a}$ and b) and faster maturation as assessed by morphology and CCR3/CD62L expression (Figures 8c and d). Again, no differences in IL-5R expression were detectable (Supplementary Figures S6a and b).

To address a possible role of GATA-2 in the observed phenotype, we assessed the expression of GATA-2, as well as that of GATA-1 and PU.1 in the bone marrow of WT and $\mathrm{RhoH}^{-1}$ mice. While GATA-1 and PU.1 levels were unchanged, GATA-2 mRNA and protein expression were reduced in the absence of $\mathrm{RhoH}$ (Figures $8 \mathrm{e}$ and $\mathrm{f}$ ), suggesting that $\mathrm{RhoH}$ also regulates GATA-2 levels in vivo.

Lack of RhoH is associated with increased numbers of differentiating eosinophils entering the cell cycle. An increased number of eosinophils in the absence of $\mathrm{RhoH}$ could be the result of decreased cell death during differentiation or increased proliferation. As induction of GATA-2 has been reported to affect cell cycling and proliferation of HPC by increasing their quiescence (G0) residency, ${ }^{10}$ we examined cell cycle phase distribution and viability of freshly isolated bone marrow cells and during eosinophil differentiation in vitro. While we did not observe any differences in viability (Supplementary Figure S6c), we found that $\mathrm{RhoH}$ deficiency did have an effect on the cell cycle (Figure 9). Using a staining protocol with 7-AAD and pyronin $\mathrm{Y}$, which allows the discrimination of cells in $\mathrm{G} 1$ from cells in $\mathrm{G} 0$, as 
a

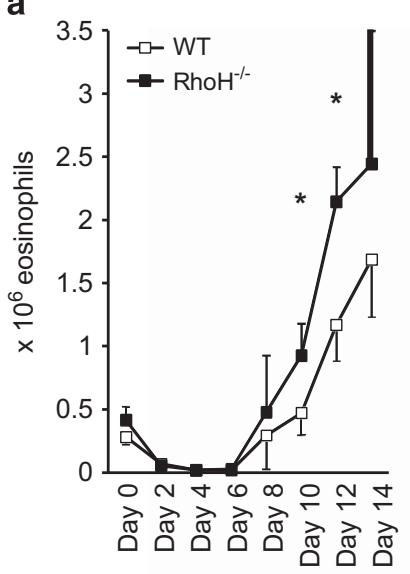

b

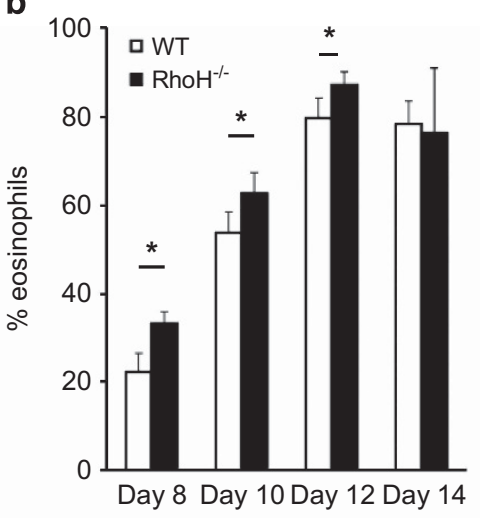

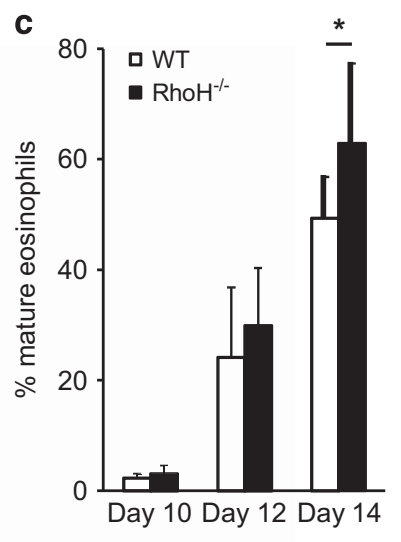
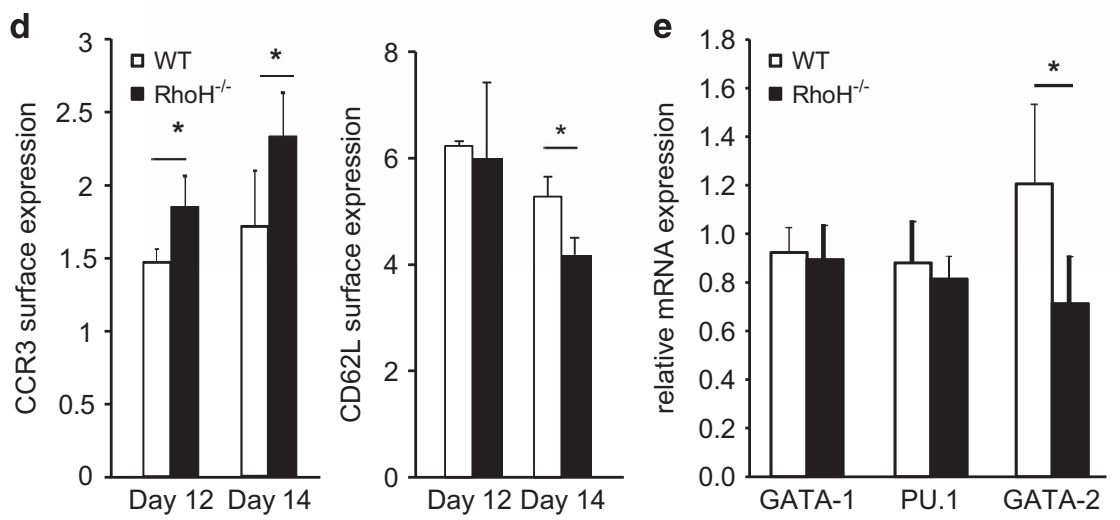

$\mathbf{f}$

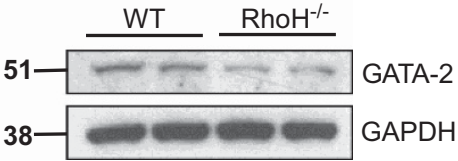

Figure 8 Enhanced eosinophil differentiation in the absence of RhoH. Bone marrow was cultured in the presence of cytokines, and cell numbers and differentials determined every second day by counting and microscopic analysis of cytospins stained with Diff-Quik. Total number (a) and percentage (b) of eosinophils from bone marrow of WT and $\mathrm{RhOH}^{-1-}$ mice. (c) Mature eosinophils as percentage of total eosinophils in culture, demonstrating faster maturation of $\mathrm{RhoH}^{-1-}$ eosinophils. Results are representative of three independent experiments with 3-4 mice per group. (d) CCR3 and CD62L expression on in vitro differentiated eosinophils on days 12 and 14 as determined by flow cytometry. (e) GATA-1, PU.1, and GATA-2 mRNA expression in freshly isolated bone marrow $(n=4)$. (f) Immunoblot analysis of GATA-2 protein expression in freshly isolated bone marrow. ${ }^{\star} P<0.05$

well as $\mathrm{S}$ and $\mathrm{G} 2 / \mathrm{M}$ phases, we found that in the absence of $\mathrm{RhoH}$, the percentage of quiescent cells having withdrawn from the cell cycle (G0) was reduced, while the percentage of cells in G1 was increased (Figure 9a). These alterations were analogous to those that have been reported for cells with increased GATA-2 levels. ${ }^{10}$ We also observed a small, nonsignificant increase in cells in $S$ phase, but no effect on $\mathrm{G} 2 / \mathrm{M}$ was apparent. As quiescent cells in G0 do not proliferate, the shift from G0 to $G 1$ and possibly $S$ indicates increased proliferation of progenitors as the mechanism underlying enhanced eosinophil generation when $\mathrm{RhoH}$ is lacking. On the other hand, when RhoH is present, a larger fraction of cells was quiescent (i.e., in G0). In order to see whether this phenomenon was detectable only at the onset of eosinophil differentiation or during the entire process, we performed the analysis each second day of the differentiation protocol (Figure 9b). The shift from G0 to G1 (and possibly S) in the absence of $\mathrm{RhoH}$ was largely maintained during eosinophil differentiation until day 10 (Figure 9b). These results suggest that $\mathrm{RhoH}$ limits eosinophil differentiation through its effect on the cell cycle, that is, by increasing quiescence (G0 residency). As RhoH induces GATA-2 (Figures 3 and 7) and GATA-2 induction has been reported to have the same effect, ${ }^{10}$ our results are consistent with $\mathrm{RhoH}$ limiting eosinophil differentiation by this mechanism.

\section{Discussion}

Our results identify $\mathrm{RhoH}$ as a novel negative regulator of eosinophil differentiation. Reduced eosinophil numbers in the presence of RhoH appear to be due to limited differentiation of eosinophils in the bone marrow rather than enhanced death of eosinophils as has recently been reported for the negative regulator PIR-A. ${ }^{39}$ Furthermore, RhoH could induce GATA-2, whereas GATA-2 expression was reduced in vivo in the absence of RhoH. RhoH limited eosinophil differentiation and maturation both in vitro and in vivo, likely by regulating GATA-2 levels and the cell cycle.

$\mathrm{RhoH}$ appears to have different functions in different hematopoietic cells. Although our data show that eosinophil differentiation is enhanced in the absence of $\mathrm{RhoH}$, T-cell development is impaired and mast cell development unaffected. ${ }^{21,26}$ Neutrophil numbers are also unchanged, probably due to the fact that they express little or no $\mathrm{RhoH}$. This observed enhancement results in increased peripheral eosinophil numbers, but the function of mature eosinophils 


\section{Bone marrow}
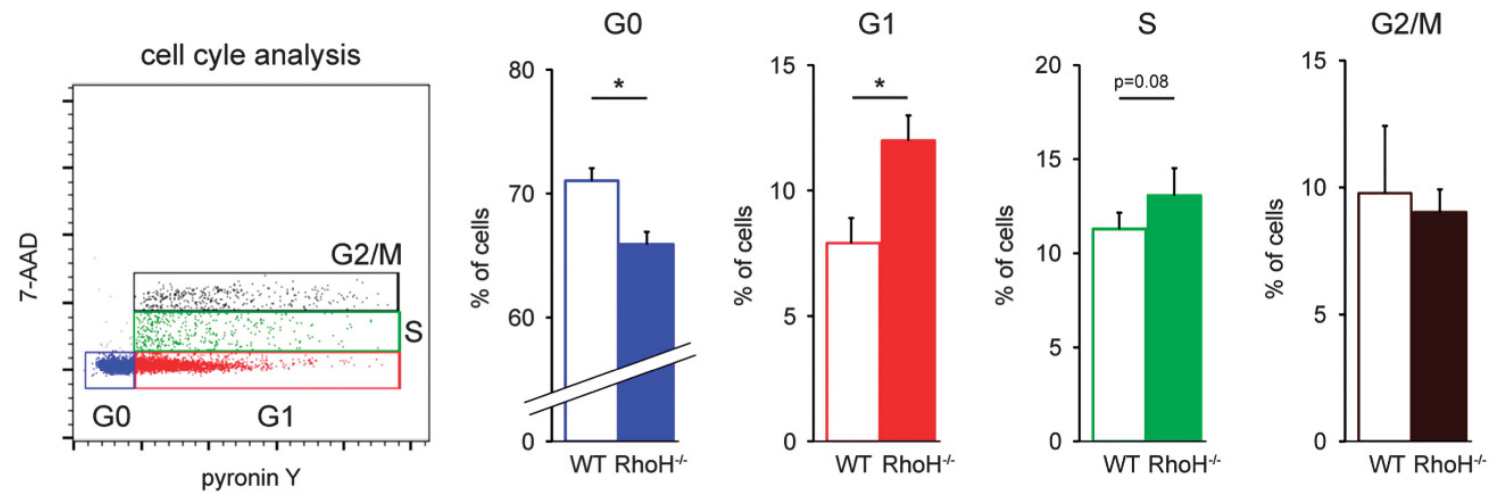

b

In vitro eosinophil differentiation

G0
G1

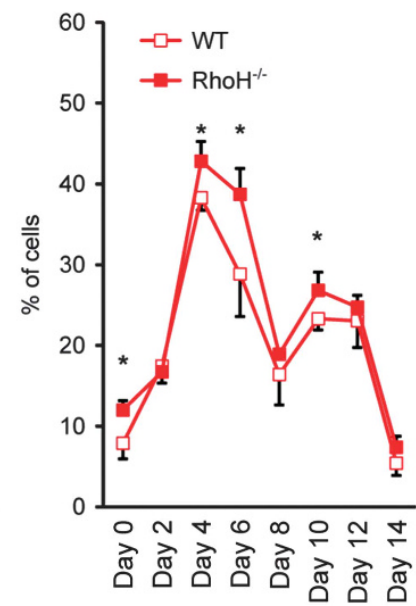

$\mathrm{S}$

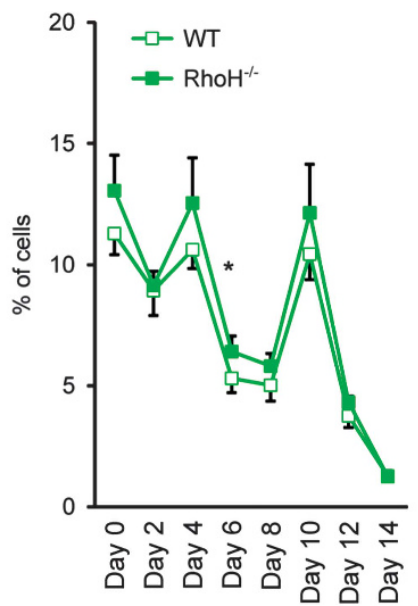

G2/M

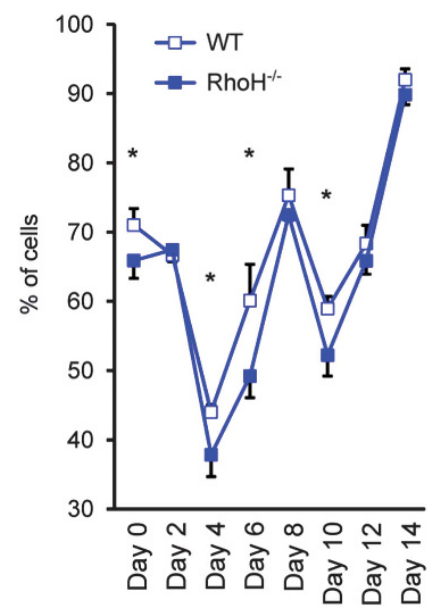

Figure 9 Cell cycle analysis in differentiating eosinophils. (a) Freshly isolated bone marrow was stained with 7-AAD (DNA) and pyronin $Y$ (RNA) to determine cell cycle distribution in WTand $\mathrm{RhOH}^{-/}$mice. Left: FACS gating strategy of stained cells. Right panels: Percentages of cells residing in the different stages of the cell cycle (G0, G1, S and G2/M) in WT and $\mathrm{RhoH}^{-1-}$ mouse bone marrow. (b) Cell cycle analysis in bone marrow cultures during eosinophil differentiation. Samples were drawn every 2 days of the differentiation protocol and analyzed as in panel (a). Values represent means $+/-$ S.D. $(n=4) .{ }^{*} P<0.05$

released into the periphery is apparently not impacted, as indicated by the results using eosinophils from $I L-5^{t g}$ and $R h o H^{-1} / L-5^{t g}$ mice. Similarly, surface expression of the maturation markers CD62L and CCR3 on mature peripheral blood eosinophils from these mice (Figure 2) or from WT and $\mathrm{RhoH}^{-1}$ mice did not differ between genotypes (not shown). The observation that differences in maturation markers are restricted to the bone marrow supports the idea that increased peripheral blood eosinophil numbers in the absence of $\mathrm{RhoH}$ are the consequence of increased eosinophil production in the bone marrow rather than enhanced release of immature eosinophils in these mice.

HPC proliferation, survival, engraftment and migration have been reported to be increased by knocking down $\mathrm{RhoH},{ }^{27}$ and our results using bone marrow chimeras of $\mathrm{RhoH}$ overexpressing and control transduced progenitors/stem cells confirm these results. Interestingly, mature eosinophil survival or migration was unaffected in our study. However, our results support the idea that $\mathrm{RhoH}$ can negatively regulate proliferation of eosinophils during differentiation, by reducing GATA-2 levels and inducing a shift from G1 to the G0 phase of cell cycle.

Reports on the role of GATA-2 in eosinophil differentiation and function have shown that it can have positive, as well as negative regulatory functions. ${ }^{8-14}$ As GATA-2 cooperates and competes with a number of transcription factors, these apparently contrasting results are likely due to differences in experimental systems and corresponding differences in the relative levels of GATA-2 and its interaction partners or competitors. Our results indicate that a partial reduction in physiological GATA-2 levels as a result of $\mathrm{RhoH}$ deficiency promotes differentiation into the eosinophil lineage. Importantly, GATA-1 levels were unaffected in $\mathrm{RhoH}^{-1}$ mice, suggesting that a shift in the GATA-1/GATA-2 balance could alleviate GATA-2 antagonism of GATA-1-mediated gene expression during eosinophil development.

Interestingly, we found that ROCK activity was required for RhoH-induced GATA-2 upregulation. ROCK is a downstream effector of several Rho proteins and has critical roles in cell cycle progression, cell survival and proliferation. ${ }^{40}$ Although 
RhoH has so far not been described to affect ROCK activity, it has been reported to antagonize Rac, and Rac can block Rho/ROCK signalling. ${ }^{27,40}$ Whether RhoH affects ROCK directly, or indirectly, for example, through relieving Racmediated antagonism of ROCK, remains to be investigated. Although ROCK has been shown to be important for cell cycle progression, it appears to affect the $\mathrm{G} 1$ to $\mathrm{S}$ transition rather than, like GATA-2, the G0 to $\mathrm{G} 1$ transition. ${ }^{41}$ Our results are, therefore, more compatible with the notion that ROCK-induced GATA-2 expression, rather than ROCK activity per se, is involved in the observed phenotype.

Besides its role in eosinophil differentiation, RhoH likely also has a role in mature eosinophils, as it is strongly and rapidly upregulated upon cytokine exposure in vitro and highly expressed in some HES patients, probably as a consequence of in vivo cytokine exposure. This rapid increase in $\mathrm{RhoH}$ protein might be facilitated by the comparatively high levels of mRNA stored in eosinophils (Figure 1a). In neutrophils, $\mathrm{RhoH}$ has been reported to limit cytokine-induced LTB4 production. ${ }^{29}$ Although this suggests that in mature eosinophils, RhoH could also act as a negative feedback regulator of cytokine signalling, we found no evidence for differences in IL-5-mediated survival or eotaxin-induced migration between mouse eosinophils with or without $\mathrm{RhoH}$ expression.

As eosinophils play major roles in asthma and allergy, eosinophil-directed therapies are currently being tested. ${ }^{18}$ While many of these successfully reduce peripheral blood eosinophil counts, they fail to eradicate tissue eosinophils. Therefore, treatments targeting de novo generation in the bone marrow and, therefore, long-term depletion might be promising. However, development of such treatments is clearly hampered by a lack of understanding of eosinophilopoiesis. For most of the genes found to be upregulated in the microarray analysis, a role in granulopoiesis/eosinophilopoiesis has not been investigated so far. As very few factors governing eosinophil generation and maturation are known (and even fewer negative regulators), and as $\mathrm{RhoH}$ negatively regulates eosinophil differentiation, our data warrant further studies to elucidate the exact molecular mechanisms underlying these processes.

The notion that $\mathrm{RhoH}$ influences transcription factors governing leukocyte differentiation is also highly interesting in a slightly different context. RhoH is frequently mutated in lymphomas and leukemias and mutations often correlate with prognosis, either poor or favorable, depending on the specific malignancy, precluding a role for $\mathrm{RhoH}$ as classical tumor suppressor or oncogene. ${ }^{19}$ The molecular basis for these apparently contradictory observations is unclear. However, shifts in the balance of transcription factors related to hematopoietic differentiation have been implicated in the pathogenesis of leukemia and therapeutic agents affecting that balance are used successfully in the clinic (e.g., all-trans retinoic acid for acute myeloid leukemia). ${ }^{42} \mathrm{~A}$ clearer view of how this network is regulated might also open up new possibilities for interventions to treat hematopoietic malignancies.

\section{Materials and Methods}

Antibodies and flow cytometry. Details are provided in the Supplementary Information.
Leukocyte isolation and culture from human blood and bone marrow. Leukocyte subsets were isolated from Li-heparinized blood as outlined in the Supplementary Information. For some experiments, eosinophils were cultured in RPMI $5 \%$ FCS with or without $10 \mathrm{ng} / \mathrm{ml} \mathrm{IL-5,} \mathrm{IL-3} \mathrm{(R \& D} \mathrm{Systems,} \mathrm{Minneapolis,}$ MN, USA), or GM-CSF (Novartis, Basel, Switzerland, Leukomax 300).

HPC and immature myeloid precursors were isolated from human bone marrow as previously described. ${ }^{43}$ Precursors were differentiated with $25 \mathrm{ng} / \mathrm{ml}$ G-CSF (neutrophils) or $10 \mathrm{ng} / \mathrm{ml} \mathrm{IL-5}$ (eosinophils). For details, see Supplementary Information.

Generation and culture of stably transduced HL-60 clone 15 cells. For generation of cell lines inducibly overexpressing RhoH see Supplementary Information. For inhibitor experiments, cells were pre-treated with SB772077B dihydrochloride ( $1 \mu \mathrm{M}$, Tocris Bioscience, Bristol, UK), PD169316, LY294002 (both $10 \mu \mathrm{M}$, Calbiochem, Darmstadt, Germany), PD98059 (50 $\mu \mathrm{M}$, Calbiochem) or FIPI hydrochloride ( $1 \mu \mathrm{M}, \mathrm{R} \& \mathrm{D}$ Systems) 45 min before induction with $100 \mathrm{nM} 4-\mathrm{OHT}$.

RT-qPCR, microarray and bioinformatics analysis. pPCR or microarray analysis using the Affimetrix Human Gene 2.0 ST Array (Affimetrix, Santa Clara, CA, USA) were performed as outlined in the Supplementary Information.

Mice and isolation of mouse eosinophils. Animal studies were approved by the Veterinary Office of the Canton of Bern and conducted in accordance with Swiss federal legislation on animal welfare. $\mathrm{RhOH}^{-1}$ mice ${ }^{24}$ were provided by Cord Brakebusch (University of Copenhagen, Denmark) and WT C57/B6 mice by the in-house facility. For functional tests, eosinophils were isolated from IL-5 ${ }^{\text {tg }}$ (C57BL/6J-Tg(II5)1638Jlee), ${ }^{16}$ provided by Jamie Lee (Mayo Clinic, Scottsdale, Arizona), and $\mathrm{RhoH}^{-1} / \mathrm{L}-5^{\text {tg }}$ mice, which were generated by crossing IL-5 $5^{\text {tg }}$ mice to $\mathrm{RhOH}^{-/}$mice. For eosinophil isolation, spleens were mechanically dissociated and RBC lysed with ACK buffer. T cells, B cells and macrophages were depleted with antibodies against $\mathrm{CD} 8 \alpha, \mathrm{CD} 90.2$ and $\mathrm{CD} 19$ (Miltenyi Biotec, Bergisch Gladbach, Germany) to obtain eosinophils ( $>90 \%$ pure by Diff-Quik).

Differentiation of eosinophils from bone marrow of 12-24-week-old mice was performed as described. ${ }^{38}$ Briefly, bone marrow of age- and sex-matched mice was cultured in the presence of Flt3L and SCF (Peprotech) for 4 days, and subsequently with IL-5 (R\&D systems) for 10 days with medium replacement every 2 days.

Bone marrow chimera. To generate mixed bone marrow chimeras, mouse bone marrow HSPCs from CD45.1 mice were transduced with lentiviral vector expressing full-length mouse RhoH (see Supplementary Information). IL-5 $5^{\text {tg }}(n=3)$ and $\mathrm{GFP}^{+} / L-5^{t g}(n=4)$ recipient mice (both $\left.\mathrm{CD} 45.2\right)$ were lethally irradiated with 10 Gy from a ${ }^{137} \mathrm{Cs}$ source (Gammacell 40 Exactor, Theratronics, Ottawa, Ontario, Canada). A $1: 1$ mixture of CD45.1 and CD45.2 transduced cells $\left(2 \times 10^{5}\right)$ were injected into the retroorbital venous sinus of recipient mice $18 \mathrm{~h}$ after irradiation. IL-5 $5^{\text {tg }}$ mice received a mixture of CD45.1 and GFP'CD45.2 HSPCs, whereas GFP $^{+} / L-5^{t g}$ mice received a mixture of $\mathrm{CD} 45.1$ and GFP neg CD45.2 HSPCs. Antibiotics (1\% Cotrim; Spirig Pharma AG, Egerkingen, Switzerland) were added to the drinking water for 4 weeks. Leukocyte subsets in blood were analyzed 4 and 8 weeks after transfer. Reconstitution efficiency was evaluated by flow cytometry analysis of recipient cells (CD45.1, CD45.2 or GFP).

\section{Conflict of Interest}

The authors declare no conflict of interest.

Acknowledgements. We wish to thank Jamie Lee and Cord Brakebusch for providing mice, John Silke for lentiviral constructs, Benjamin Gantenbein for providing human bone marrow, Kevin Oberson for experimental animal support and flow cytometry, staff of the GTF Lausanne for support with microarray data analysis, and doctors, patients and donors for providing blood samples. This study was supported by grants of the Swiss National Science Foundation (grant no. 310030_146181 and 310030_166473 to HUS and 310030_749790 to CB), Deutsche Forschungsgemeinschaft (grant no. STO-906/1-1 to CS) and Novartis Stiftung für medizinischbiologische Forschung (grant no. 14B074 to CS).

1. Rosenberg HF, Dyer KD, Foster PS. Eosinophils: changing perspectives in health and disease. Nat Rev Immunol 2013; 13: 9-22. 
2. Soragni A, Yousefi S, Stoeckle C, Soriaga AB, Sawaya MR, Kozlowski E et al. Toxicity of eosinophil MBP is repressed by intracellular crystallization and promoted by extracellular aggregation. Mol Cell 2015; 57: 1011-1021.

3. Simon D, Simon HU. Eosinophilic disorders. J Allergy Clin Immunol 2007; 119: 1291-1300.

4. Valent $P$, Klion $A D$, Horny HP, Roufosse F, Gotlib J, Weller PF et al. Contemporary consensus proposal on criteria and classification of eosinophilic disorders and related syndromes. J Allergy Clin Immunol 2012; 130: 607-612.

5. Lammel V, Stoeckle C, Padberg B, Zweifel R, Kienle DL, Reinhart WH et al. Hypereosinophilia driven by GM-CSF in large-cell carcinoma of the lung. Lung Cancer 2012; 76: 493-495.

6. Rothenberg ME, Hogan SP. The eosinophil. Annu Rev Immunol 2006; 24: 147-174.

7. McNagny K., Graf T. Making eosinophils through subtle shifts in transcription factor expression. J Exp Med 2002; 195: F43-F47.

8. Tsai FY, Keller G, Kuo FC, Weiss M, Chen J, Rosenblatt M et al. An early haematopoietic defect in mice lacking the transcription factor GATA-2. Nature 1994; 371: 221-226.

9. Persons DA, Allay JA, Allay ER, Ashmun RA, Orlic D, Jane SM et al. Enforced expression of the GATA-2 transcription factor blocks normal hematopoiesis. Blood 1999; 93 488-499.

10. Tipping AJ, Pina C, Castor A, Hong D, Rodrigues NP, Lazzari L et al. High GATA-2 expression inhibits human hematopoietic stem and progenitor cell function by effects on cell cycle. Blood 2009; 113: 2661-2672.

11. Iwasaki H, Mizuno S, Arinobu $\mathrm{Y}$, Ozawa $\mathrm{H}$, Mori $\mathrm{Y}$, Shigematsu $\mathrm{H}$ et al. The order of expression of transcription factors directs hierarchical specification of hematopoietic lineages. Genes Dev 2006; 20: 3010-3021.

12. Qiu Z, Dyer KD, Xie Z, Radinger M, Rosenberg HF. GATA transcription factors regulate the expression of the human eosinophil-derived neurotoxin (RNase 2) gene. J Biol Chem 2009; 284: 13099-13109.

13. Yamaguchi $Y$, Ackerman SJ, Minegishi N, Takiguchi M, Yamamoto M, Suda T. Mechanisms of transcription in eosinophils: GATA-1, but not GATA-2, transactivates the promoter of the eosinophil granule major basic protein gene. Blood 1998; 91: 3447-3458.

14. Yang D, Suzuki S, Hao LJ, Fujii Y, Yamauchi A, Yamamoto $M$ et al. Eosinophil-specific regulation of gp91(phox) gene expression by transcription factors GATA-1 and GATA-2. J Bio Chem 2000; 275: 9425-9432.

15. Geering B, Stoeckle C, Conus S, Simon HU. Living and dying for inflammation: neutrophils, eosinophils, basophils. Trends Immunol 2013; 34: 398-409.

16. Lee NA, McGarry MP, Larson KA, Horton MA, Kristensen AB, Lee JJ. Expression of IL-5 in thymocytes/T cells leads to the development of a massive eosinophilia, extramedullary eosinophilopoiesis, and unique histopathologies. J Immunol 1997; 158: 1332-1344.

17. Stoeckle C, Simon HU. CD8+ T cells producing IL-3 and IL-5 in non-lgE-mediated eosinophilic diseases. Allergy 2013; 68: 1622-1625.

18. Radonjic-Hoesli S, Valent P, Klion AD, Wechsler ME, Simon HU. Novel targeted therapies for eosinophil-associated diseases and allergy. Annu Rev Pharmacol Toxicol 2015; 55: 633-656.

19. Troeger A, Williams DA. Hematopoietic-specific Rho GTPases Rac2 and RhoH and human blood disorders. Exp Cell Res 2013; 319: 2375-2383.

20. Chae HD, Siefring JE, Hildeman DA, Gu Y, Williams DA. RhoH regulates subcellular localization of ZAP-70 and Lck in T cell receptor signaling. PLoS One 2010; 5: e13970.

21. Gu Y, Chae HD, Siefring JE, Jasti AC, Hildeman DA, Williams DA. RhoH GTPase recruits and activates Zap70 required for $T$ cell receptor signaling and thymocyte development. Nat Immunol 2006; 7: 1182-1190.

22. Wang $\mathrm{H}$, Zeng $\mathrm{X}$, Fan Z, Lim B. RhoH modulates pre-TCR and TCR signalling by regulating LCK. Cell Signal 2011; 23: 249-258.

23. Schmidt-Mende J, Geering B, Yousefi S, Simon HU. Lysosomal degradation of RhoH protein upon antigen receptor activation in T but not B cells. Eur J Immunol 2010; 40 $525-529$.
24. Dorn T, Kuhn U, Bungartz G, Stiller S, Bauer M, Ellwart J et al. RhoH is important for positive thymocyte selection and T-cell receptor signaling. Blood 2007; 109: 2346-2355.

25. Crequer A, Troeger A, Patin E, Ma CS, Picard C, Pedergnana V et al. Human $\mathrm{RHOH}$ deficiency causes T cell defects and susceptibility to EV-HPV infections. J Clin Invest 2012; 122: 3239-3247.

26. Oda $H$, Fujimoto M, Patrick MS, Chida D, Sato $Y$, Azuma $Y$ et al. RhoH plays critical roles in Fc epsilon RI-dependent signal transduction in mast cells. J Immunol 2009; 182: 957-962.

27. Gu Y, Jasti AC, Jansen M, Siefring JE. RhoH, a hematopoietic-specific Rho GTPase, regulates proliferation, survival, migration, and engraftment of hematopoietic progenitor cells. Blood 2005; 105: 1467-1475.

28. Gundogdu MS, Liu H, Metzdorf D, Hildebrand D, Aigner M, Aktories $\mathrm{K}$ et al. The haematopoietic GTPase RhoH modulates IL3 signalling through regulation of STAT activity and IL3 receptor expression. Mol Cancer 2010; 9: 225.

29. Daryadel A, Yousefi S, Troi D, Schmid I, Schmidt-Mende J, Mordasini C et al. RhoH/TTF negatively regulates leukotriene production in neutrophils. J Immunol 2009; 182: 6527-6532.

30. Geering B, Schmidt-Mende J, Federzoni E, Stoeckle C, Simon HU. Protein overexpression following lentiviral infection of primary mature neutrophils is due to pseudotransduction. J Immunol Methods 2011; 373: 209-218.

31. Xu W, Wang P, Petri B, Zhang Y, Tang W, Sun L et al. Integrin-induced PIP5K1C kinase polarization regulates neutrophil polarization, directionality, and in vivo infiltration. Immunity 2010; 33: 340-350.

32. Tamassia N, Bazzoni F, Le Moigne V, Calzetti F, Masala C, Grisendi G et al. IFN-beta expression is directly activated in human neutrophils transfected with plasmid DNA and is further increased via TLR-4-mediated signaling. J Immunol 2012; 189: 1500-1509.

33. Vince JE, Wong WW, Khan N, Feltham R, Chau D, Ahmed AU et al. IAP antagonists target CIAP1 to induce TNFalpha-dependent apoptosis. Cell 2007; 131: 682-693.

34. Voehringer D, van Rooijen N, Locksley RM. Eosinophils develop in distinct stages and are recruited to peripheral sites by alternatively activated macrophages. J Leukoc Biol 2007; 81: 1434-1444.

35. Waterstrat A, Liang Y, Swiderski CF, Shelton BJ, van Zant G. Congenic interval of CD $45 / L y-5$ congenic mice contains multiple genes that may influence hematopoietic stem cell engraftment. Blood 2010; 115: 408-417.

36. Basu S, Ray A, Dittel BN. Differential representation of B cell subsets in mixed bone marrow chimera mice due to expression of allelic variants of CD45. J Immunol Methods 2013; 396: 163-167.

37. Mercier FE, Sykes DB, Scadden DT. Single targeted exon mutation creates a true congenic mouse for competitive hematopoietic stem cell transplantation: The C57BL/6-CD45.1STEM mouse. Stem Cell Reports 2016; 6: 985-992.

38. Dyer KD, Moser JM, Czapiga M, Siegel SJ, Percopo CM, Rosenberg HF. Functionally competent eosinophils differentiated ex vivo in high purity from normal mouse bone marrow. J Immunol 2008; 181: 4004-4009.

39. Ben Baruch-Morgenstern N, Shik D, Moshkovits I, Itan M, Karo-Atar D, Bouffi C et al. Paired immunoglobulin-like receptor $\mathrm{A}$ is an intrinsic, self-limiting suppressor of IL-5-induced eosinophil development. Nat Immunol 2014; 15: 36-44.

40. Amin E, Dubey BN, Zhang SC, Gremer L, Dvorsky R, Moll JM et al. Rho-kinase: regulation, (dys)function, and inhibition. Biol Chem 2013; 394: 1399-1410.

41. Zhang S, Tang Q, Xu F, Xue Y, Zhen Z, Deng Y et al. RhoA regulates G1-S progression of gastric cancer cells by modulation of multiple INK4 family tumor suppressors. Mol Cancer Res 2009; 7: 570-580.

42. Martelli MP, Gionfriddo I, Mezzasoma F, Milano F, Pierangeli S, Mulas F et al. Arsenic trioxide and all-trans retinoic acid target NPM1 mutant oncoprotein levels and induce apoptosis in NPM1-mutated AML cells. Blood 2015; 125: 3455-3465.

43. Geering B, Stoeckle C, Rozman S, Oberson K, Benarafa C, Simon HU. DAPK2 positively regulates motility of neutrophils and eosinophils in response to intermediary chemoattractants. J Leukoc Biol 2014; 95: 293-303.

\section{Supplementary Information accompanies this paper on Cell Death and Differentiation website (http://www.nature.com/cdd)}

